\title{
Association between polymorphisms of prokineticin receptor (PKR1 rs4627609 and PKR2 rs6053283) and recurrent pregnancy loss*
}

\author{
Yun-lei $\mathrm{CAO}^{1}$, Zhao-feng $\mathrm{ZHANG}^{2}$, Jian $\mathrm{WANG}^{2}$, Mao-hua MIAO ${ }^{2}$, Jian-hua XU' ${ }^{2}$, \\ Yue-ping $\mathrm{SHEN}^{3}$, Ai-min $\mathrm{CHEN}^{4}$, Jing DU ${ }^{\dagger \downarrow 2}$, Wei YUAN ${ }^{\dagger 2}$ \\ ('Shanghai Obstetrics/Gynecology Hospital, Fudan University, Shanghai 200011, China) \\ ( ${ }^{2}$ Key Laboratory of Reproduction Regulation of NPFPC, SIPPR, IRD, Fudan University, Shanghai 200032, China) \\ ( ${ }^{3}$ Department of Biostatistics and Epidemiology, Public Health School, Soochow University, Suzhou 215123, China) \\ ( ${ }^{4}$ Division of Epidemiology and Biostatistics, Department of Environmental Health, \\ University of Cincinnati College of Medicine, Cincinnati, OH 45267, USA) \\ †E-mail: dujing42@126.com; yuanwei11@yahoo.com \\ Received July 25, 2015; Revision accepted Dec. 3, 2015; Crosschecked Feb. 15, 2016
}

\begin{abstract}
Recurrent pregnancy loss (RPL) is a condition with complex etiologies, to which both genetic and environmental factors may contribute. During the last decade, studies indicated that the expression patterns of the prokineticin receptor (PKR1 and PKR2) are closely related to early pregnancy. However, there are few studies on the role of PKR1 and PKR2 in RPL. In this study, we purpose to investigate the association between polymorphisms of the prokineticin receptor (PKR1 rs4627609 and PKR2 rs6053283) and RPL on a group of 93 RPL cases and 169 healthy controls. Genotyping of the single nucleotide polymorphisms (SNPs) was performed using a Sequenom MassARRAY iPLEX system. The results revealed a significant association between PKR2 rs6053283 polymorphism and RPL $(P=0.003)$, whereas no association was observed between PKR1 rs4627609 polymorphism and RPL $(P=0.929)$ in the Chinese Han population.
\end{abstract}

Key words: Prokineticin receptor 1 (PKR1), PKR2, Polymorphism, Recurrent pregnancy loss http://dx.doi.org/10.1631/jzus.B1500180 CLC number: R714.21

\section{Introduction}

Recurrent pregnancy loss (RPL) is defined as resulting in at least two consecutive idiopathic miscarriages before 20 weeks of gestation by the Practice Committee of the American Society for Reproductive Medicine (2013). Approximately $2 \%-4 \%$ of the women of childbearing age are suffering from this problem (Li et al., 2001). The causes of RPL are complicated: chromosome abnormalities, anatomical abnormalities, endocrinology disorders, immune

\footnotetext{
¿ Corresponding authors

* Project supported by the National Natural Science Foundation of China (No. 81571503)

(D) ORCID: Jing DU, http://orcid.org/0000-0002-6767-2763

(C) Zhejiang University and Springer-Verlag Berlin Heidelberg 2016
}

disorders, thrombophilia, maternal infections, and nutritional deficiencies whicn are the most common etiologies; however, $50 \%$ of RPLs with no obvious causes are known as unexplained RPLs (Li et al., 2002).

Prokineticins (PKs) are recently discovered to be tissue-specific angiogenesis factors, comprising of PK1 and PK2, which are also known as endocrine gland-derived vascular endothelial growth factors (EG-VEGFs) and Bv8. In humans, EG-VEGF and Bv8 with $83 \%$ amino-acid identity, play the same role and possess the same $G$ protein-coupled receptors, and are defined as prokineticin receptor 1 (PKR1) and PKR2 (Lin et al., 2002; Le Couter et al., 2003). PK1 expression is primarily localized in the steroidogenic glands including the ovary, testis, adrenal gland, and 
placenta (Le Couter et al., 2001). The distribution of PK2 expression is similar to PK1, but is found to be at the highest level in testis and peripheral blood leukocytes (Li et al., 2001). Le Couter et al. (2001) have briefly reported PK1 expression in the placenta. Hoffmann et al. (2006) have recently characterized PK1 expression in human placenta during the early period of pregnancy and suggested that PK1, but not PK2, expresses in human placenta and is primarily located in the syncytiotrophoblast layer, during the 8 to 10 weeks of gestation, and the expression of PK1 emerges as the highest level and can be up-regulated by hypoxia. PK1 can promote angiogenesis via inducing tissue-specific proliferation, migration and fenestration in capillary endothelial cells, and the function of angiogenesis seems to be limited to endothelial cells derived from endocrine tissues (Cheng et al., 2002). Furthermore, microarray analysis revealed that PK1 may participate in regulating the expression of multiple cytokines and implantation of endometrium (Evans et al., 2008; Haouzi et al., 2009). PKs play a role through their receptors (PKRs). As a result of binding with PKs, PKRs couple to Gq proteins, and then promote intracellular $\mathrm{Ca}^{2+}$ mobilization (Lin et al., 2002; Soga et al., 2002). In addition, PKRs can also couple to $\mathrm{Gi}$ and Gs proteins, indicating that PKRs are found to be involved in activating multiple intracellular signal-transduction pathways (Chen et al., 2005). Activation of PKRs influences several physiological events, such as intestinal contraction, spermatogenesis, circadian rhythm, hematopoiesis, and angiogenesis (Schweitz et al., 1999; Wechselberger et al., 1999; Le Couter et al., 2001; 2002; 2003; 2004; Morton et al., 2005).

Given the potential regulation of the PKs/PKRs pathways in human early pregnancies, we hypothesized that polymorphisms of PKRs are involved in abnormal angiogenesis and implantation failure. The present study aims to investigate the association between the polymorphisms of PKRs (PKR1 rs4627609 and PKR2 rs6053283) and RPL.

\section{Materials and methods}

\subsection{Subjects}

The study was approved by the Ethics Committee of Soochow University (Suzhou, China). A total of 93 RPL cases (mean \pm standard deviation (SD) age,
(28.30 \pm 3.77$)$ years; body mass index (BMI), $(20.93 \pm$ $\left.2.23) \mathrm{kg} / \mathrm{m}^{2}\right)$ and 169 ethnic-matched healthy controls (mean \pm SD age, $(28.08 \pm 3.62)$ years; BMI, $(20.10 \pm$ 2.29) $\mathrm{kg} / \mathrm{m}^{2}$ ) were included in this study. Patients aged 20-40 years with two or more consecutive unexplained terminations of pregnancy before 12 gestational weeks or expulsions of a fetus weighing $<500 \mathrm{~g}$ were identified and recruited when they visited the Maternal and Child Health Center in Kunshan City, the First People's Hospital and the Second People's Hospital which are affiliated with Soochow University for repeated treatment (two or more times). All patients with RPL had a naturally conceived pregnancy. Subjects with anatomical abnormality, chromosome abnormality, endocrine disorder, autoimmune disease, or coagulopathy were excluded from the study. One hundred and sixty-nine age-matched pregnant women with at least one naturally conceived pregnancy and no history of miscarriage were recruited as control subjects ( 2 for each case patient). None of the controls had a history of pregnancy complications, still birth, pre-eclampsia, or giving birth to a child who was small for its gestational age. The matching criteria included age ( \pm 2 years), gestational age ( \pm 1 week), and residence (the same district).

Information on demographic characteristics, lifestyle, obstetric history, and other RPL risk factors was collected using a structured questionnaire. Anticoagulant peripheral blood samples were obtained from all the participants during the following two weeks after their day of recruitment, and stored at $-80{ }^{\circ} \mathrm{C}$ until the genomic DNA was isolated.

\subsection{Genotyping}

Genomic DNA was extracted from the lymphocyte of the anticoagulant peripheral blood using the QIA-amp DNA blood kit (QIAGEN, Hilden, Germany) according to the manufactures' instructions. The quality evaluation of the extracted DNA was performed using a NanoDrop spectrophotometer (ThermoScientific) by measuring the absorbance at $260 \mathrm{~nm}$. The DNA sample used for genotyping is $30 \mathrm{ng} / \mu \mathrm{l}$ with an $\mathrm{OD}_{260} / \mathrm{OD}_{280}$ (OD: optical density) range from 1.8 to 2.0 and an $\mathrm{OD}_{260} / \mathrm{OD}_{230}$ range of no less than 1.5 .

The single nucleotide polymorphisms (SNPs) of rs4627609 and rs6053283 were detected by using a Sequenom MassARRAY iPLEX platform (Sequenom, San Diego, CA, USA), in which a $90 \%$ sample quality 
control (QC) rate and a 90\% SNP genotyping success rate were imposed. Briefly, a single base extension, following an initial locus-specific PCR reaction, was conducted by using mass-modified dideoxynucleotide terminators of an oligonucleotide primer which immediately anneals upstream to the polymorphic site of interest, and then, via using a matrix-assisted laser desorption and ionization time-of-flight (MALDITOF) mass spectrometry, the SNP allele was identified by the distinct mass of the extended primer (Gabriel et al., 2009).

\subsection{Statistical analysis}

The Hardy-Weinberg equilibrium and the comparisons of genotype and allele frequencies between RPL cases and the controls were performed using the online software SHEsis to perform a Chi-square test (http://analysis.bio-X.cn/myAnalysis.php) (Shi and He, 2005). Using the SPSS Version 13.0, a univariate logistic regression was conducted, and then adjusted by the BMI of the participants. The independentsamples $t$-test between the RPL cases and controls on the women's age and BMI showed that there was a significant difference between the case and control groups on BMI $(P=0.005$; Table 1). The adjusted odds ratios (AORs) and their $95 \%$ confidence intervals (CIs) were used to measure the strength of the association between the RPL and polymorphisms of rs4627609 and rs6053283. In order to account for multiple testing, significance levels were adjusted by the Bonferroni correction, which was made for 2 SNPs, and the significance level was reduced to 0.025 $(0.05 / 2)$, where appropriate (O'Callaghan et al., 2012).
Table 1 Independent-samples $t$-test between case and control subjects

\begin{tabular}{lcc}
\hline \multicolumn{1}{c}{ Group } & Age (year) & BMI $\left(\mathrm{kg} / \mathrm{m}^{2}\right)$ \\
\hline Case $(n=93)$ & $28.301 \pm 3.767$ & $20.926 \pm 2.226$ \\
Control $(n=169)$ & $28.083 \pm 3.618$ & $20.103 \pm 2.289$ \\
\hline$P$-value & 0.650 & 0.005 \\
\hline
\end{tabular}

Data are expressed as mean $\pm \mathrm{SD}$. Age: age of the participants; BMI: body mass index of the participants

\subsection{Prediction of SNP effects}

The prediction of the SNP effects on mRNA splicing was performed using the web-based prediction software ESEfinder (http://rulai.cshl.edu/cgi-bin/ tools/ESE3/esefinder.cgi?process=home) (Smith et al., 2006).

\section{Results}

The genotype distributions of the two polymorphisms both in the case and control groups were all within the Hardy-Weinberg equilibrium $(P>0.05)$. The data of genotype and allele frequencies are shown in Tables 2 and 3. The results indicated that the distribution of the genotype and allele frequencies of PKR2 rs6053283 showed significant differences between the RPL cases and the healthy controls $(P=0.009$ and $P=0.003$, respectively). The univariate logistic regression analyses showed that women with rs6053283 AA genotype had a higher risk of RPL than GG genotype carriers (AOR: 2.65, 95\% CI: $1.14-6.17, P=0.024$ ) and $A$ allele was positively associated with the increased risk of RPL (AOR: 2.14, 95\% CI: 1.28-3.58,

Table 2 Genotype and allele frequencies of the PKR1 rs4627609 SNP in RPL patients and controls

\begin{tabular}{|c|c|c|c|c|c|c|c|c|c|}
\hline \multirow{2}{*}{ SNP } & \multirow{2}{*}{ Group } & \multicolumn{4}{|c|}{ Genotype frequency } & \multicolumn{4}{|c|}{ Allele frequency } \\
\hline & & TT & $\mathrm{TC}$ & $\mathrm{CC}$ & $P$-value & $\mathrm{T}$ & $\mathrm{C}$ & $P$-value & OR $(95 \% \mathrm{CI})$ \\
\hline PKR1 & Case & $38(0.409)$ & $37(0.398)$ & $18(0.194)$ & 0.714 & $113(0.608)$ & $73(0.392)$ & 0.929 & $0.98(0.68-1.42)$ \\
\hline (rs4627609) & Control & $64(0.379)$ & $76(0.450)$ & $29(0.172)$ & & $204(0.604)$ & $134(0.396)$ & & \\
\hline
\end{tabular}

Data are expressed as number (frequency)

Table 3 Genotype and allele frequencies of the PKR2 rs6053283 SNP in RPL patients and controls

\begin{tabular}{|c|c|c|c|c|c|c|c|c|c|}
\hline \multirow{2}{*}{ SNP } & \multirow{2}{*}{ Group } & \multicolumn{4}{|c|}{ Genotype frequency } & \multicolumn{4}{|c|}{ Allele frequency } \\
\hline & & GG & GA & AA & $P$-value & G & A & $P$-value & OR $(95 \% \mathrm{CI})$ \\
\hline PKR2 & Case & $63(0.677)$ & $25(0.269)$ & $5(0.054)$ & 0.009 & $151(0.812)$ & $35(0.188)$ & 0.003 & $2.14(1.28-3.58)$ \\
\hline (rs6053283) & Control & $137(0.811)$ & $31(0.183)$ & $1(0.006)$ & & $305(0.902)$ & $33(0.098)$ & & \\
\hline
\end{tabular}

Data are expressed as number (frequency) 
Table 4 Univariate logistic regression for dependent variables: having RPL according to genotype distributions of investigated SNPs as independent variables

\begin{tabular}{|c|c|c|c|c|}
\hline \multirow{2}{*}{ Genotype and allele } & \multicolumn{2}{|c|}{ Number (frequency) } & \multirow{2}{*}{$\operatorname{AOR}(95 \% \mathrm{CI})$} & \multirow{2}{*}{$P$-value } \\
\hline & Case $(n=93)$ & Control $(n=169)$ & & \\
\hline \multicolumn{5}{|l|}{ PKR1 rs4627609 } \\
\hline TT & $38(0.409)$ & $64(0.379)$ & 1.00 (ref) & \\
\hline $\mathrm{TC}$ & $37(0.398)$ & $76(0.450)$ & $1.20(0.68-2.14)$ & 0.527 \\
\hline $\mathrm{CC}$ & $18(0.194)$ & $29(0.172)$ & $0.94(0.66-1.36)$ & 0.746 \\
\hline $\mathrm{TC}+\mathrm{CC}$ & & & $1.10(0.65-1.87)$ & 0.718 \\
\hline $\mathrm{T}$ & $113(0.608)$ & $204(0.604)$ & 1.00 (ref) & \\
\hline $\mathrm{C}$ & $73(0.392)$ & $134(0.396)$ & $0.98(0.68-1.42)$ & 0.929 \\
\hline \multicolumn{5}{|l|}{ PKR2 rs6053283 } \\
\hline GG & $63(0.677)$ & $137(0.811)$ & $1.00(\mathrm{ref})$ & \\
\hline GA & $25(0.269)$ & $31(0.183)$ & $1.64(0.88-3.06)^{\#}$ & 0.122 \\
\hline AA & $5(0.054)$ & $1(0.006)$ & $2.65(1.14-6.17)$ & 0.024 \\
\hline $\mathrm{GA}+\mathrm{AA}$ & & & $1.94(1.07-3.50)$ & 0.028 \\
\hline $\mathrm{G}$ & $151(0.812)$ & $305(0.902)$ & 1.00 (ref) & \\
\hline A & $35(0.188)$ & $33(0.098)$ & $2.14(1.28-3.58)$ & 0.003 \\
\hline
\end{tabular}

AOR: adjusted odd ratio by body mass index of female participants; ref: reference group. ${ }^{*}$ Significance level $\alpha=0.025$ by Bonferroni correction

$P=0.003$ ) (Table 4); however the polymorphism of rs4627609 was not associated with RPL.

The wild-type (G) allele and mutant-type (A) allele of PKR2 rs6053283 were analyzed using ESEfinder, and the data indicated that the mutant-type "A" allele can affect the splicing regulation by altering the exonic splicing enhancer (ESE) motifs. The results showed that the mutant-type "A" results in a gain within the SC35 motif (1.12) (Fig. 1). Serine/ arginine-rich (SR) proteins, which have distinct functions in alternative pre-mRNA splicing, are bound to ESEs and function as general activators of the exon definition. The SR protein family members include SF2/ASF, SC35, SRp20, SRp40, SRp55, SRp75, SRp30c, 9G8, and SRp54 (Long and Caceres, 2009).

\section{Discussion}

The results of the independent-samples $t$-test between the RPL case and the control groups on women's ages and BMI revealed that there were significant differences between the case and control groups in BMI $(P=0.005)$. In order to reduce the influence of the confounding factors, the OR was adjusted by the BMI for all the subjects. Although, the AOR was estimated as a measure of the strength of the association between the polymorphisms and RPL, it was still difficult to rule out other confounding factors, such as the living environments and dietary

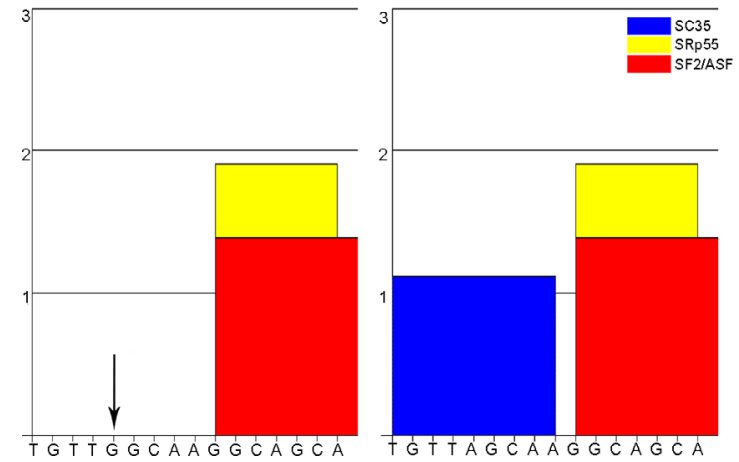

Fig. 1 Prediction for binding of splicing enhancer: an outcome of rs6053283 mutation

The wild-type "G" and mutant-type "A" alleles of rs6053283 were predicted by ESEfinder to disrupt ESE sites of one or more of four different SR proteins (SF2/ASF (red), SC35 (blue), SRp55 (yellow)), and the results are shown in the top panels. Colored bars represent individual ESE sites with the matrix score (higher score represents a stronger prediction) plotted on the vertical axis and the position of the ESE within each rs6053283 represented on the horizontal axis (Note: for interpretation of the references to color in this figure legend, the reader is referred to the web version of this article)

habits. Even so, the results demonstrated a strong association between the polymorphism of PKR2 rs6053283 and RPL ( $P=0.003)$.

The beginning and maintenance of a successful pregnancy depends on many factors, such as angiogenesis and endometrial receptivity. Angiogenesis is very important in the process of placental vessel formation and maternal vascular adaptation. PK1 and its receptors (PKR1, PKR2), are shown to be expressing in the human placenta of the first and third 
trimester periods and locating to the syncytiotrophoblast layer, cytotrophoblast layer, fetal endothelium, and macrophages, which mediates tissue-specific angiogenesis, immune regulation, and modulation of the inflammatory responses (Le Couter et al., 2001; Dorsch et al., 2005; Hoffmann et al., 2006; Denison et al., 2008). All of the above functions play critical roles in endometrial receptivity, embryo implantation, and placentation. Meanwhile, the expression pattern of PK1 and its receptor indicates their essential roles in early human pregnancy. The expression of PK1 and PKR1 reaches its peak during the hypoxic period of the placental development. Reports suggested that the dynamic expression of PK1 during the pregnancy period may possibly be regulated by up-regulated hormones during early pregnancy (estrogen, progesterone, and human chorionic gonadotrophin) (Battersby et al., 2004; Evans et al., 2009; Shaw et al., 2010). PK1 and VEGF have similar biological functions during human pregnancy (Le Couter et al., 2003). Both of them are expressed in human placenta; the difference lies in the fact that the VEGF expression is restricted to the cytotrophoblasts and extravillous trophoblasts (Hoffmann et al., 2006), and polymorphisms of the VEGF have been associated with the risk of RPL (Lee et al., 2010). Given these facts, we speculated that PK1 and VEGF perform complementary roles during pregnancy.

Evidence shows that PK1 expression is elevated during the implantation period (Evans et al., 2008). PK1 was shown to stimulate a group of genes, including interleukin 11 (IL-11), IL-6, leukemia inhibitory factor $(L I F)$, and cyclooxygenase $2(C O X-2)$, which play important roles in embryo implantation and decidualization (Stewart et al., 1992; Tabibzadeh et al., 1995; Lim et al., 1997; Robb et al., 1998; Denison et al., 2008; Haouzi et al., 2009). PK1 can induce its target genes by phosphorylation of their downstream target extracellular signal-regulated kinase $1 / 2$ through cross-talk with the epidermal growth factor receptor system, or by activation of mitogenactivated protein kinase and the PI3 kinase/Akt pathway (Lin et al., 2002; Haouzi et al., 2009). Denison et al. (2008) suggested that PK1 facilitated the embryo implantation via inducing the $L I F$ expression. Recently, PK1 was reported as a uterine receptivity marker (Evans et al., 2008). All of the evidence unanimously revealed the important role of PK1 in embryo implantation. Therefore, we surmised that the polymorphisms of PK1 and its receptors may be associated with RPL.

The mutation of PKR2 has been reported to be associated with hypogonadotropic hypogonadism. Kallmann syndrome patients with biallelic PKR2 mutations had a severe reproductive phenotype. Biallelic PKR2 mutations have also been reported in women suffering from primary amenorrhea and breast development dysfunction, especially in monoallelic mutation of PKR2 carriers (Monnier et al., 2009; Sarfati et al., 2010). Other disorders associated with the PK1 signaling pathway include polycystic ovarian syndrome (PCOS), ectopic pregnancy, endometriosis, and pre-eclampsia (Hoffmann et al., 2006; Shaw et al., 2010; Tiberi et al., 2010). Studies have demonstrated that the PK1 expression is higher in ectopic endometrial and polycystic ovarian tissue in patients with endometriosis or PCOS, and this suggests the role of angiogenesis of PK1 (Ferrara et al., 2003; Shaw et al., 2010). The association between polymorphisms of PK1 and its receptors with RPL was first studied in the Taiwanese Han population. Su et al. (2010) found that PKR1 rs4627609 and PKR2 rs6053283 polymorphisms were associated with idiopathic RPL. This study was conducted in the East China Han population, and further confirmed the association between PKR2 rs6053283 and RPL. However, we failed to discover the association between PKR1 rs4627609 and RPL. This inconsistent finding may primarily be attributed to the small samples both in our study and previous reports. Moreover, the differences of geographic environments between Taiwanese and East China may also have an influence on the results. To a certain extent, environmental factors play a key role in the occurrence of RPL as well.

In conclusion, our findings indicated that polymorphism in PKR2 rs6053283 was obviously associated with idiopathic RPL and could be developed as a new biomarker to evaluate the risk of RPL. However, there is no study performed in other populations and the underlying mechanism of PKR2 rs6053283 polymorphism in the development of RPL is largely unknown, so further studies are warranted to replicate our findings in different ethnic groups with larger sample sizes. It is also necessary to validate the function of the PKR2 rs6053283 polymorphism on RPL. 


\section{Acknowledgements}

We would like to thank all the participants for providing their blood samples. This study was conducted at the NPFPC Laboratory of Contraception and Devices, Shanghai Institute of Planned Parenthood Research, Shanghai 200032, China.

\section{Compliance with ethics guidelines}

Yun-lei CAO, Zhao-feng ZHANG, Jian WANG, Mao-hua MIAO, Jian-hua XU, Yue-ping SHEN, Ai-min CHEN, Jing DU, and Wei YUAN declare that they have no conflict of interest.

All procedures followed were in accordance with the ethical standards of the responsible committee on human experimentation (institutional and national) and with the Helsinki Declaration of 1975, as revised in 2008 (5). Informed consent was obtained from all patients for being included in the study.

\section{References}

Battersby, S., Critchley, H.O., Morgan, K., et al., 2004. Expression and regulation of the prokineticins (endocrine gland-derived vascular endothelial growth factor and Bv8) and their receptors in the human endometrium across the menstrual cycle. J. Clin. Endocrinol. Metab., 89(5):24632469. http://dx.doi.org/10.1210/jc.2003-032012

Chen, J., Kuei, C., Sutton, S., et al., 2005. Identification and pharmacological characterization of prokineticin $2 \beta$ as a selective ligand for prokineticin receptor 1. Mol. Pharmacol., 67(6):2070-2076. http://dx.doi.org/10.1124/mol.105.011619

Cheng, M.Y., Bullock, C.M., Li, C., et al., 2002. Prokineticin 2 transmits the behavioural circadian rhythm of the suprachiasmatic nucleus. Nature, 417(6887):405-410. http://dx.doi.org/10.1038/417405a

Denison, F.C., Battersby, S., King, A.E., et al., 2008. Prokineticin-1: a novel mediator of the inflammatory response in third-trimester human placenta. Endocrinology, 149(7):3470-3477. http://dx.doi.org/10.1210/en.2007-1695

Dorsch, M., Qiu, Y., Soler, D., et al., 2005. PK1/EG-VEGF induces monocyte differentiation and activation. J. Leukoc. Biol., 78(2):426-434. http://dx.doi.org/10.1189/jlb.0205061

Evans, J., Catalano, R.D., Morgan, K., et al., 2008. Prokineticin 1 signaling and gene regulation in early human pregnancy. Endocrinology, 149(6):2877-2887. http://dx.doi.org/10.1210/en.2007-1633

Evans, J., Catalano, R.D., Brown, P., et al., 2009. Prokineticin 1 mediates fetal-maternal dialogue regulating endometrial leukemia inhibitory factor. FASEB J., 23(7):2165-2175. http://dx.doi.org/10.1096/fj.08-124495

Ferrara, N., Frantz, G., Le Couter, J., et al., 2003. Differential expression of the angiogenic factor genes vascular endothelial growth factor (VEGF) and endocrine glandderived VEGF in normal and polycystic human ovaries.
Am. J. Pathol., 162(6):1881-1893.

http://dx.doi.org/10.1016/S0002-9440(10)64322-2

Gabriel, S., Ziaugra, L., Tabbaa, D., 2009. SNP genotyping using the Sequenom MassARRAY iPLEX platform. Curr. Protoc. Hum. Genet., 60:2.12.1-2.12.18. http://dx.doi.org/10.1002/0471142905.hg0212s60

Haouzi, D., Mahmoud, K., Fourar, M., et al., 2009. Identification of new biomarkers of human endometrial receptivity in the natural cycle. Hum. Reprod., 24(1):198-205. http://dx.doi.org/10.1093/humrep/den360

Hoffmann, P., Feige, J.J., Alfaidy, N., 2006. Expression and oxygen regulation of endocrine gland-derived vascular endothelial growth factor/prokineticin-1 and its receptors in human placenta during early pregnancy. Endocrinology, 147(4):1675-1684. http://dx.doi.org/10.1210/en.2005-0912

Le Couter, J., Kowalski, J., Foster, J., et al., 2001. Identification of an angiogenic mitogen selective for endocrine gland endothelium. Nature, 412(6850):877-884. http://dx.doi.org/10.1038/35091000

Le Couter, J., Lin, R., Ferrara, N., 2002. Endocrine glandderived VEGF and the emerging hypothesis of organspecific regulation of angiogenesis. Nat. Med., 8(9):913-917. http://dx.doi.org/10.1038/nm0902-913

Le Couter, J., Lin, R., Tejada, M., et al., 2003. The endocrinegland-derived VEGF homologue Bv8 promotes angiogenesis in the testis: localization of Bv8 receptors to endothelial cells. PNAS, 100(5):2685-2690.

http://dx.doi.org/10.1073/pnas.0337667100

Le Couter, J., Zlot, C., Tejada, M., et al., 2004. Bv8 and endocrine gland-derived vascular endothelial growth factor stimulate hematopoiesis and hematopoietic cell mobilization. PNAS, 101(48):16813-16818. http://dx.doi.org/10.1073/pnas.0407697101

Lee, H.H., Hong, S.H., Shin, S.J., et al., 2010. Association study of vascular endothelial growth factor polymorphisms with the risk of recurrent spontaneous abortion. Fertil. Steril., 93(4):1244-1247. http://dx.doi.org/10.1016/j.fertnstert.2008.11.017

Li, M., Bullock, C.M., Knauer, D.J., et al., 2001. Identification of two prokineticin cDNAs: recombinant proteins potently contract gastrointestinal smooth muscle. Mol. Pharmacol., 59(4):692-698. http://dx.doi.org/10.1124/mol.59.4.692

Li, T.C., Makris, M., Tomsu, M., et al., 2002. Recurrent miscarriage: aetiology, management and prognosis. Hum. Reprod. Update, 8(5):463-481. http://dx.doi.org/10.1093/humupd/8.5.463

Lim, H., Paria, B.C., Das, S.K., et al., 1997. Multiple female reproductive failures in cyclooxygenase 2-deficient mice. Cell, 91(2):197-208. http://dx.doi.org/10.1016/S0092-8674(00)80402-X

Lin, D.C., Bullock, C.M., Ehlert, F.J., et al., 2002. Identification and molecular characterization of two closely related G protein-coupled receptors activated by prokineticins/ endocrine gland vascular endothelial growth factor. $J$. 
Biol. Chem., 277(22):19276-19280.

http://dx.doi.org/10.1074/jbc.M202139200

Long, J.C., Caceres, J.F., 2009. The SR protein family of splicing factors: master regulators of gene expression. Biochem. J., 417(1):15-27.

http://dx.doi.org/10.1042/BJ20081501

Monnier, C., Dode, C., Fabre, L., et al., 2009. PROKR2 missense mutations associated with Kallmann syndrome impair receptor signalling activity. Hum. Mol. Genet., 18(1):75-81. http://dx.doi.org/10.1093/hmg/ddn318

Morton, A.J., Wood, N.I., Hastings, M.H., et al., 2005. Disintegration of the sleep-wake cycle and circadian timing in Huntington's disease. J. Neurosci., 25(1):157-163. http://dx.doi.org/10.1523/JNEUROSCI.3842-04.2005

O'Callaghan, M.E., MacLennan, A.H., Gibson, C.S., et al., 2012. Fetal and maternal candidate single nucleotide polymorphism associations with cerebral palsy: a case-control study. Pediatrics, 129(2):e414-e423. http://dx.doi.org/10.1542/peds.2011-0739

Practice Committee of the American Society for Reproductive Medicine, 2013. Definitions of infertility and recurrent pregnancy loss: a committee opinion. Fertil. Steril., 99(1):63. http://dx.doi.org/10.1016/j.fertnstert.2012.09.023

Robb, L., Li, R., Hartley, L., et al., 1998. Infertility in female mice lacking the receptor for interleukin 11 is due to a defective uterine response to implantation. Nat. Med., 4(3):303-308. http://dx.doi.org/10.1038/nm0398-303

Sarfati, J., Guiochon-Mantel, A., Rondard, P., et al., 2010. A comparative phenotypic study of Kallmann syndrome patients carrying monoallelic and biallelic mutations in the prokineticin 2 or prokineticin receptor 2 genes. J. Clin. Endocrinol. Metab., 95(2):659-669. http://dx.doi.org/10.1210/jc.2009-0843

Schweitz, H., Pacaud, P., Diochot, S., et al., 1999. MIT 1 , a black mamba toxin with a new and highly potent activity on intestinal contraction. FEBS Lett., 461(3):183-188. http://dx.doi.org/10.1016/S0014-5793(99)01459-3

Shaw, J.L., Denison, F.C., Evans, J., et al., 2010. Evidence of prokineticin dysregulation in fallopian tube from women with ectopic pregnancy. Fertil. Steril., 94(5):1601-1608. http://dx.doi.org/10.1016/j.fertnstert.2009.10.061

Shi, Y.Y., He, L., 2005. SHEsis, a powerful software platform for analyses of linkage disequilibrium, haplotype construction, and genetic association at polymorphism loci. Cell Res., 15(2):97-98. http://dx.doi.org/10.1038/sj.cr.7290272

Smith, P.J., Zhang, C., Wang, J., et al., 2006. An increased specificity score matrix for the prediction of SF2/ASFspecific exonic splicing enhancers. Hum. Mol. Genet., 15(16):2490-2508. http://dx.doi.org/10.1093/hmg/ddl171
Soga, T., Matsumoto, S., Oda, T., et al., 2002. Molecular cloning and characterization of prokineticin receptors. Biochim. Biophys. Acta, 1579(2-3):173-179. http://dx.doi.org/10.1016/S0167-4781(02)00546-8

Stewart, C.L., Kaspar, P., Brunet, L.J., et al., 1992. Blastocyst implantation depends on maternal expression of leukaemia inhibitory factor. Nature, 359(6390):76-79. http://dx.doi.org/10.1038/359076a0

Su, M.T., Lin, S.H., Lee, I.W., 2010. Polymorphisms of endocrine gland-derived vascular endothelial growth factor gene and its receptor genes are associated with recurrent pregnancy loss. Hum. Reprod., 25(11):2923-2930. http://dx.doi.org/10.1093/humrep/deq256

Tabibzadeh, S., Kong, Q.F., Babaknia, A., et al., 1995. Progressive rise in the expression of interleukin-6 in human endometrium during menstrual cycle is initiated during the implantation window. Hum. Reprod., 10(10):2793-2799.

Tiberi, F., Tropea, A., Apa, R., et al., 2010. Prokineticin 1 mRNA expression in the endometrium of healthy women and in the eutopic endometrium of women with endometriosis. Fertil. Steril., 93(7):2145-2149. http://dx.doi.org/10.1016/j.fertnstert.2009.01.105

Wechselberger, C., Puglisi, R., Engel, E., et al., 1999. The mammalian homologues of frog Bv8 are mainly expressed in spermatocytes. FEBS Lett., 462(1-2):177-181. http://dx.doi.org/10.1016/S0014-5793(99)01473-8

\section{中文概要}

题 目: 前动力蛋白受体 (PKR1 rs4627609 和 PKR2 rs6053283）的多态性与复发性流产的关系

目 的: 研究前动力蛋白受体 PKR1 rs4627609 和 PKR2 rs6053283 的多态性与复发性流产的关系, 并对 其可能的作用机制进行预测。

创新点: 首次在中国汉族人群中进行前动力蛋白受体 PKR1 rs4627609 和 PKR2 rs6053283 的多态性与 复发性流产关系的研究, 并对其功能进行预测。

方 法: 共收集了 93 例复发性流产和 169 例健康对照者 血液样本, 提取基因组 DNA, 对 PKR1 rs4627609 和 PKR2 rs6053283 两个位点进行基因多态性分 析, 在两组样本中分析不同基因型与复发性流产 的关系, 并对 PKR2 rs6053283 位点的不同等位 基因进行生物功能预测。

结 论: PKR2 rs6053283 的多态性与复发性流产相关; 而 PKR1 rs4627609 的多态性与复发性流产之间不存 在相关性。

关键词: 前动力蛋白受体 1 ; 前动力蛋白受体 2 ; 多态性; 复发性流产 